\title{
Evaluation of the Ribosomal Protein S1 Gene (rpsA) as a Novel Biomarker for Mycobacterium Species Identification
}

\author{
Hongfei Duan, Guan Liu, Xiaobo Wang, Yuhong Fu, Qian Liang, Yuanyuan Shang, \\ Naihui Chu, and Hairong Huang
}

\begin{abstract}
National Clinical Laboratory on Tuberculosis, Beijing Key Laboratory on Drug-Resistant Tuberculosis, Beijing Chest Hospital, Capital Medical University, Beijing Tuberculosis \& Thoracic Tumor Research Institute, 97 Beimachang, Tongzhou Qu, Beijing 101149, China

Correspondence should be addressed to Naihui Chu; dongchu1994@sina.com and Hairong Huang; hairong.huangcn@gmail.com
\end{abstract}

Received 26 July 2014; Accepted 7 November 2014

Academic Editor: Om Parkash

Copyright (C) 2015 Hongfei Duan et al. This is an open access article distributed under the Creative Commons Attribution License, which permits unrestricted use, distribution, and reproduction in any medium, provided the original work is properly cited.

\begin{abstract}
Objectives. To evaluate the resolution and reliability of the $\operatorname{rps} A$ gene, encoding ribosomal protein S1, as a novel biomarker for mycobacteria species identification. Methods. A segment of the rpsA gene ( $565 \mathrm{bp}$ ) was amplified by PCR from 42 mycobacterial reference strains, 172 nontuberculosis mycobacteria clinical isolates, and $16 \mathrm{M}$. tuberculosis complex clinical isolates. The PCR products were sequenced and aligned by using the multiple alignment algorithm in the MegAlign package (DNASTAR) and the MEGA program. A phylogenetic tree was constructed by the neighbor-joining method. Results. Comparative sequence analysis of the rpsA gene provided the basis for species differentiation within the genus Mycobacterium. Slow- and rapid-growing groups of mycobacteria were clearly separated, and each mycobacterial species was differentiated as a distinct entity in the phylogenetic tree. The sequences discrepancy was obvious between $M$. kansasii and M. gastri, M. chelonae and M. abscessus, M. avium and M. intracellulare, and M. szulgai and M. malmoense, which cannot be achieved by $16 \mathrm{~S}$ ribosomal DNA (rDNA) homologue genes comparison. 183 of the 188 (97.3\%) clinical isolates, consisting of 8 mycobacterial species, were identified correctly by $r p s A$ gene blast. Conclusions. Our study indicates that rpsA sequencing can be used effectively for mycobacteria species identification as a supplement to $16 \mathrm{~S}$ rDNA sequence analysis.
\end{abstract}

\section{Introduction}

Members of the genus Mycobacterium are widespread in nature and range from harmless saprophytic species to strict pathogens that cause serious human and animal diseases. Both slow-growing mycobacteria and rapid-growing mycobacteria can cause human infections. Traditionally, taxonomy based on biochemical characteristics has been used for species determination of mycobacteria, but this approach is limited due to the overlapping biochemical and phenotypic patterns among the different mycobacterial species. Another approach using analysis of cell-wall fatty acid and mycolic acid composition is also limited by profile similarity among some emerging nontuberculosis mycobacteria (NTM) [1]. 16S rDNA homologue gene sequence comparison has been used as an important method for the mycobacterial species identification; however, ambiguous results have been obtained either due to the presence of more than one copy of the $16 \mathrm{~S}$
rDNA gene within the genome, for example, in $M$. celatum and $M$. terrae complex [2,3], or due to sequence homology between species [4]. Therefore, alternative phylogenetic markers which are capable of complementing 16S rRNA gene would be useful for the phylogenetic study and species identification of the genus Mycobacterium.

Ribosomal protein $S 1$ ( $r p s A$ ), which is in the 30 S ribosome subunit, contains the $\mathrm{S} 1$ domain that has been found in a large number of RNA-associated proteins. RpsA is a vital protein involved in protein translation and the ribosome-sparing process of translation. In addition, it has been reported that RpsA is the target of pyrazinoic acid, the active form of the antituberculosis drug pyrazinamide [5]. Mycobacterium tuberculosis has a single copy of the rpsA gene in the genome [6], while the sequence homology among different mycobacteria species in which the gene has already been sequenced is between 86.7 and $100 \%$. This suggests that rpsA may be 
suitable for phylogenetic study of the genus Mycobacterium. In this paper, we report an evaluation of the $r p s A$ gene as a novel biomarker for mycobacteria species identification.

\section{Materials and Methods}

2.1. Mycobacterial Reference Strains and Clinical Isolates. 42 type and reference strains of the genus Mycobacterium (Table 1), 172 clinical NTM isolates, and $16 \mathrm{M}$. tuberculosis complex (MTC) isolates were investigated. All the type and reference strains were purchased from the American Type Culture Collection (ATCC) and all the clinical isolates used in this study were obtained from the Clinical Database and Sample Bank of tuberculosis of Beijing, National Clinical Lab on Tuberculosis, Beijing Chest Hospital. All the clinical NTM isolates were identified to the species level by sequence alignment of at least two of the following: 16S rDNA, 16$23 \mathrm{~S}$ rRNA gene internal transcribed spacer (ITS), and rpoB and hsp65 genes as described before [2, 7-9]. Following sequencing, the 172 NTM clinical strains were found to include 10 strains of $M$. avium, 75 strains of $M$. intracellulare, 23 strains of $M$. kansasii, 39 strains of $M$. abscessus, 17 strains of $M$. fortuitum complex, 7 strains of $M$. gordonae, and 1 strain of M. neoaurum.

2.2. rpsA Gene Amplification and Sequencing. DNA was released from cultured mycobacteria by boiling the cultured mycobacterial suspension in TE buffer for $10 \mathrm{~min}$. After centrifugation, the supernatant was used for PCR amplification [10]. The primers used were forward primer, $5^{\prime}$-CCCTACATCGGCAAGGAG-3', position $487-504$ in the rpsA gene of Mycobacterium tuberculosis, GenBank accession number NC_000962.2, and reverse primer, 5' -TGTCGATGACCTTGACCATC-3', position 1032-1051 in the rpsA gene of Mycobacterium tuberculosis, GenBank accession number NC_000962.2. The amplified product was $565 \mathrm{bp}$. PCR products were purified and sequenced by a commercial company (YINGJUN Biotech Company, Beijing, China) using ABI 3730 DNA Analyzer (Applied Biosystems, California, USA).

2.3. Sequence Analysis and the Phylogenetic Tree Constructions. In addition to the 42 reference strains, the rps $A$ sequences of 5 other mycobacterial species were obtained directly from GenBank, including $M$. avium subspecies paratuberculosis (GenBank accession number NC_002944.2), M. ulcerans (GenBank accession number NC_008611.1), $M$. vanbaalenii (GenBank accession number NC_008726.1), $M$. abscessus subspecies massiliense (GenBank accession number NC_018150.1), and M. canettii (GenBank accession number NC_015848.1). All sequences were aligned and homology was calculated by using the multiple alignment algorithm in the MegAlign package (Windows version 7.1.0; DNASTAR, Madison, Wis). $527 \mathrm{bp}$ of sequence (excluding 38 nucleotides at each end of the amplicon, corresponding to the primer binding sites) was analyzed for the phylogenetic tree construction by the neighbor-joining method using the MEGA 5.05 package (http://www.megasoftware.net/mega.php). A bootstrap analysis (1000 repeats) using Rhodococcus equi
(GenBank accession number NC_006361.1) as the outgroup was performed to evaluate the topology of the phylogenetic tree.

2.4. Species Identification of the Clinical Isolates. The 188 clinical isolates were analyzed blindly. Sequences, minus the known PCR primer sequences, were assembled and edited by using SeqMan software (version 7.1.0; DNASTAR, Madison, WI). Isolates were identified by comparing sequences by using a FASTA BLASTn search with MegAlign (version 7.1.0; DNASTAR, Madison, WI) to an in-house database of sequences consisting of type and reference strains from external culture collections.

\section{Results}

3.1. rpsA Sequence Alignment of the Reference Strains. Between $85.4 \%$ and $100 \%$ sequence homology (interspecies divergence, $0 \%$ to $14.6 \%$ ) was observed among the 42 tested reference strains and the 5 additional mycobacterial species whose sequences were obtained from the GenBank database (Table 2). All the rpsA gene sequences of the analyzed mycobacteria strains were distinct from the outgroup strain $R$. equi. Among the 19 reference strains of the slow-growing Mycobacterium genus, 11 strains were greater than $97 \%$ homology, including $5 \mathrm{M}$. tuberculosis complex strains. Among the 28 reference strains of the rapidgrowing Mycobacterium genus, 14 strains were greater than 97\% homology (Table 1). The pathogenic M. kansasii was easily differentiated from the nonpathogenic M. gastri $(95.8 \%$ homology), while those two species were not distinguishable by the $16 \mathrm{~S}$ rDNA sequence alignment. The sequence homologies between various species were $91.8 \%$ between $M$. chelonae and M. abscessus, $95.6 \%$ between M. avium and M. intracellulare, and $93.9 \%$ between $M$. szulgai and $M$. malmoense. However, the sequence homology between other species was higher; for example, it was $99.6 \%$ between $M$. ulcerans and M. marinum and $98.7 \%$ between $M$. abscessus subspecies massiliense and M. abscessus. All members of the $M$. tuberculosis complex had identical sequences as did $M$. senegalense and $M$. thermoresistibile, M. parafortuitum and $M$. trivial, $M$. diernhoferi and $M$. duvalii, and $M$. austroafricanum and M. terrae.

3.2. Phylogenetic Tree Construction. A phylogenetic tree, which provided the basis for species differentiation in the genus Mycobacterium, was constructed (Figure 1). The absolute majority tested species showed good separation. The rapid-growing species were well defined from the slowgrowing species in the tree. $M$. chelonae, $M$. abscessus subspecies massiliense, and M. abscessus, which are categorized in the pathogenic taxonomic group of rapid-growing mycobacteria, formed a distinctive cluster which was much closer to the slow-growing species compared with the nonpathogenic group of rapid-growing mycobacteria. The reliability of the phylogenetic tree was verified by the bootstrap method, using $R$. equi as the outgroup. 
TABLE 1: Percentage $r p s A$ sequence match among type strain.

\begin{tabular}{|c|c|c|c|}
\hline Species & Type strain & First match $(\%)^{\mathrm{a}}$ & Second match $(\%)^{\mathrm{a}}$ \\
\hline M. thermoresistibile & ATCC19527 & M. senegalense $(100 \%)$ & M. porcinum $(98.7 \%)$ \\
\hline M. senegalense & ATCC35796 & M. thermoresistibile (100\%) & M. porcinum $(98.7 \%)$ \\
\hline M. porcinum & ATCC33776 & M. senegalense/M. thermoresistibile (98.7\%) & M. fortuitum (98.3\%) \\
\hline M. fortuitum & ATCC6481 & M. porcinum $(98.3 \%)$ & $\begin{array}{l}\text { M. senegalense/M. thermoresistibile } \\
(98.1 \%)\end{array}$ \\
\hline M. phlei & ATCC11758 & M. porcinum $(96.5 \%)$ & M. fortuitum $(96.1 \%)$ \\
\hline M. gadium & ATCC27726 & M. porcinum/M. fortuitum (95.7\%) & M. smegmatis (95.3\%) \\
\hline M. flavescens & ATCC14474 & M. smegmatis $(96.6 \%)$ & M. vaccae $(96.0 \%)$ \\
\hline M. smegmatis & ATCC19420 & $\begin{array}{l}\text { M. vaccae/M. parafortuitum/M. triviale } \\
(96.6 \%)\end{array}$ & $\begin{array}{l}\text { M. senegalense/M. thermoresistibile } \\
(96.3 \%)\end{array}$ \\
\hline M. vaccae & ATCC15483 & M. diernhoferi/M. duvalii (96.8\%) & M. parafortuitum/M. triviale (96.6\%) \\
\hline M. parafortuitum & ATCC19686 & M. triviale (100\%) & M. terrae/M. austroafricanum (96.6\%) \\
\hline M. triviale & ATCC23292 & M. parafortuitum (100\%) & M. terrae/M. austroafricanum (96.6\%) \\
\hline M. neoaurum & ATCC25795 & M. diernhoferi/M. duvalii (96.4\%) & M. porcinum $(95.5 \%)$ \\
\hline M. diernhoferi & ATCC19340 & M. duvalii $(100 \%)$ & M. vaccae $(96.8 \%)$ \\
\hline M. duvalii & ATCC43910 & M. diernhoferi (100\%) & M. vaccae $(96.8 \%)$ \\
\hline M. aurum & ATCC23366 & M. gilvum $(93.9 \%)$ & M. chubuense/M. vanbaalenii (93.0\%) \\
\hline M. chubuense & ATCC27278 & M. gilvum (96.6\%) & M. vanbaalenii $(96.0 \%)$ \\
\hline M. gilvum & ATCC43909 & M. vanbaalenii (97.2\%) & M. terrae/M. austroafricanum (97.0\%) \\
\hline M. vanbaalenii & PYR-1 & M. terrae/M. austroafricanum (99.8\%) & M. gilvum (97.2\%) \\
\hline M. terrae & ATCC15755 & M. austroafricanum (100\%) & M. vanbaalenii (99.8\%) \\
\hline M. austroafricanum & ATCC33464 & M. terrae $(100 \%)$ & M. vanbaalenii (99.8\%) \\
\hline M. rhodesiae & ATCC27024 & M. terrae/M. austroafricanum (93.9\%) & M. obuense $(93.7 \%)$ \\
\hline M. obuense & ATCC27023 & M. porcinum /M. fortuitum (96.1\%) & $\begin{array}{l}\text { M. senegalense/M. thermoresistibile } \\
(96.5 \%)\end{array}$ \\
\hline M. aichiense & ATCC27280 & M. sphagni (95.8\%) & M. vaccae $(94.7 \%)$ \\
\hline M. sphagni & ATCC33027 & M. aichiense (95.8\%) & M. porcinum (95.5\%) \\
\hline M. tokaiense & ATCC27282 & M. porcinum $(95.3 \%)$ & M. smegmatis (93.7\%) \\
\hline M. chelonae & ATCC35752 & M. obuense (93.9\%) & M. phlei (93.5\%) \\
\hline $\begin{array}{l}\text { M. abscessus subspecies } \\
\text { massiliense }\end{array}$ & str. GO 06 & M. abscessus $(98.7 \%)$ & M. sphagni (91.4\%) \\
\hline M. abscessus & ATCC19977 & M. abscessus subspecies massiliense (98.7\%) & M. chubuense $(92.0 \%)$ \\
\hline M. tuberculosis $\mathrm{H} 37 \mathrm{Rv}$ & ATCC27294 & $\begin{array}{l}\text { M. tuberculosis } \mathrm{H} 37 \mathrm{Ra} / M \text {. africanum/M. } \\
\text { bovis } \mathrm{BCG} / M \text {. bovis }(100 \%)\end{array}$ & M. canettii $(99.8 \%)$ \\
\hline M. tuberculosis $\mathrm{H} 37 \mathrm{Ra}$ & ATCC25177 & $\begin{array}{l}\text { M. tuberculosis } \mathrm{H} 37 \mathrm{Rv} / M \text {. africanum/M. } \\
\text { bovis BCG/M. bovis (100\%) }\end{array}$ & M. canettii (99.8\%) \\
\hline M. africanum & ATCC25420 & $\begin{array}{l}\text { M. tuberculosis } \mathrm{H} 37 \mathrm{Ra} / M \text {. tuberculosis } \\
\text { Rv/M. bovis BCG/M. bovis (100\%) }\end{array}$ & M. canettii (99.8\%) \\
\hline M. bovis $\mathrm{BCG}$ & ATCC35735 & $\begin{array}{l}\text { M. tuberculosis } \mathrm{H} 37 \mathrm{Ra} / M . \text { tuberculosis } \\
\mathrm{H} 37 \mathrm{Rv} / M \text {. africanum/M. bovis }(100 \%)\end{array}$ & M. canettii (99.8\%) \\
\hline M. bovis & ATCC19210 & $\begin{array}{l}\text { M. tuberculosis } \mathrm{H} 37 \mathrm{Ra} / M \text {. tuberculosis } \\
\mathrm{H} 37 \mathrm{Rv} / M \text {. africanum/M. bovis BCG }(100 \%)\end{array}$ & M. canettii (99.8\%) \\
\hline M. canettii & CIPT140010059 & $\begin{array}{l}\text { M. tuberculosis/M. africanum/M. bovis } \\
\text { BCG/M. bovis }(99.8 \%)\end{array}$ & M. asiaticum (93.2\%) \\
\hline M. xenopi & ATCC19250 & M. asiaticum (93.0\%) & $\begin{array}{l}\text { M. avium subspecies paratuberculosis } \\
(92.4 \%)\end{array}$ \\
\hline M. avium & ATCC25291 & $\begin{array}{l}\text { M. avium subspecies paratuberculosis } \\
(99.6 \%)\end{array}$ & M. asiaticum (95.8\%) \\
\hline
\end{tabular}


TABle 1: Continued.

\begin{tabular}{|c|c|c|c|}
\hline Species & Type strain & First match $(\%)^{\mathrm{a}}$ & Second match $(\%)^{\mathrm{a}}$ \\
\hline $\begin{array}{l}\text { M. avium subspecies } \\
\text { paratuberculosis }\end{array}$ & $\mathrm{K}-10$ & M. $\operatorname{avium}(99.4 \%)$ & M. asiaticum (96\%) \\
\hline M. malmoense & ATCC29571 & M. intracellulare (94.3\%) & M. szulgai $(93.9 \%)$ \\
\hline M. intracellulare & ATCC13950 & M. $\operatorname{avium}(95.6 \%)$ & $\begin{array}{l}\text { M. avium subspecies paratuberculosis } \\
(95.4 \%)\end{array}$ \\
\hline M. scrofulaceum & ATCC19981 & M. asiaticum (95.4\%) & M. szulgai $(95.1 \%)$ \\
\hline M. marinum & ATCC927 & M. ulcerans $(99.6 \%)$ & M. szulgai (96.0\%) \\
\hline M. ulcerans & Agy99 & M. marinum $(99.6 \%)$ & M. szulgai $(96.0 \%)$ \\
\hline M. szulgai & ATCC25799 & M. marinum/M. ulcerans $(96.0 \%)$ & M. asiaticum (95.6\%) \\
\hline M. gordonae & ATCC14470 & M. asiaticum $(95.3 \%)$ & M. kansasii (95.1\%) \\
\hline M. asiaticum & ATCC25276 & M. simiae $(97.7 \%)$ & M. kansasii (96.4\%) \\
\hline M. kansasii & ATCC12478 & M. asiaticum $(96.3 \%)$ & M. gastri $(95.8 \%)$ \\
\hline M. gastri & ATCC15754 & M. kansasii (95.8\%) & M. asiaticum $(94.5 \%)$ \\
\hline Rhodococcus equi & $103 \mathrm{~S}$ & M. smegmatis $(90.3 \%)$ & M. intracellulare $(90.1 \%)$ \\
\hline
\end{tabular}

${ }^{a}$ Beside the queried type strain itself, the first matched or the second matched type strain.

3.3. Species Identification Outcomes of the Clinical Isolates. rps A sequence and alignment efficiently identified clinical isolates representing 8 mycobacterial species. We found that a criterion of first distinct $r$ psA sequence match $>97 \%$ confirmed the identification of 183 of 188 (97.3\%) clinical isolates. $20 \mathrm{M}$. abscessus strains, $2 \mathrm{M}$. fortuitum strains, and $10 \mathrm{M}$. avium strains were identified to the subspecies level. Identification discrepancies between $\operatorname{rps} A$ and other sequences were only encountered with $4 M$. gordonae strains and $1 M$. kansasii strain (see Table 3). No discrepancies were found in the species submitted as M. intracellulare, M. avium, M. abscessus, and M. tuberculosis complex.

The sequence divergence among MTC members was $0.4 \%$. The intraspecies divergence among the 188 clinical strains ranged from $0.6 \%$ to $5.3 \%$ (Table 3 ). The sequence diversity among the $M$. abscessus was $0.6 \%$, while that among the M. gordonae clinical isolates was 5.3\%. No M. gordonae clinical strain had an identical sequence with that of the M. gordonae reference strain (ATCC14470). Interestingly, the rpsA sequence of one strain among the $23 \mathrm{M}$. kansasii isolates exhibited a relatively low level of sequence similarity (95.6\%) to that of the reference strain (ATCC 12478), while those of all the other $M$. kansasii isolates were identical. Since the strain was confirmed as $M$. kansasii by rpoB and $h s p 65$ gene alignment, it might suggest a distant variant or a new subtype.

\section{Discussion}

$16 \mathrm{~S}$ rDNA gene sequence alignment has been used as the reference method for mycobacterial species identification. However, it has been reported that, by using the $16 \mathrm{~S}$ rDNA gene alone for species identification of clinical NTM, 37\% of such isolates remained unclassified which illustrates the need for additional molecular tools for proper phylogenetic assignment and accurate NTM identification [11]. The common assumption that bacterial isolates belong to the same species if they have fewer than $5-15$ bp differences within the 16S rDNA gene sequence [12] or if they have more than 97\% 16S rDNA gene sequence identities [13] may not be applicable to genus of Mycobacterium, whose members are much more closely related to each other. Furthermore, some mycobacterial strains shared uniform sequence of $16 \mathrm{~S}$ rDNA gene, such as $M$. intracellulare and $M$. avium, M. kansasii and M. gastri, M. abscessus and M. chelonae, and M. szulgai and M. malmoense.

In this study, we found that the rpsA gene is useful as a complementary method to the $16 \mathrm{~S}$ rDNA for mycobacterial species identification. Among the 47 reference strains and totally 1081 paired comparisons $\left\{1081=\left[n a_{1}+n(n-1) d / 2\right]\right.$; $\left.a_{1}=1, n=46, d=1\right\}$, the rpsA gene alone can differentiate $97.2 \%$ (1051 out of 1081) of them if using $97 \%$ sequence homology as cutoff value. When referring to the phylogenetic tree, the rpsA resolution might increase. For the most commonly seen pathogenic NTM species such as $M$. intracellulare, $M$. avium, $M$. abscessus, $M$. chelonae, and $M$. xenopi, rpsA gene sequence could differentiate these species easily. Furthermore, 183 of the 188 (97.3\%) clinical isolates, representing 8 mycobacterial species, were identified correctly by rpsA gene blast. Discrepancies of identification were only encountered with $4 \mathrm{M}$. gordonae strains and 1 M. kansasii strain. Both these species have reportedly more intraspecies sequence divergence $[9,14-16]$. Three out of the 4 M. gordonae strains with discrepant identification still had the first distinction as M. gordonae by $r p s A$, but the sequence identity was lower than $97 \%$, which means the identification might be correct when a more complete in-house database is being developed.

rpsA alone cannot differentiate the following: between members of the M. tuberculosis complex, between M. senegalense and $M$. thermoresistibile, between $M$. parafortuitum and M. trivial, between M. diernhoferi and M. duvalii, and between $M$. austroafricanum and $M$. terrae; however, these species are also difficult to be separated by other markers alone such as 16s rDNA, ITS, rpoB, and hsp65. Several 


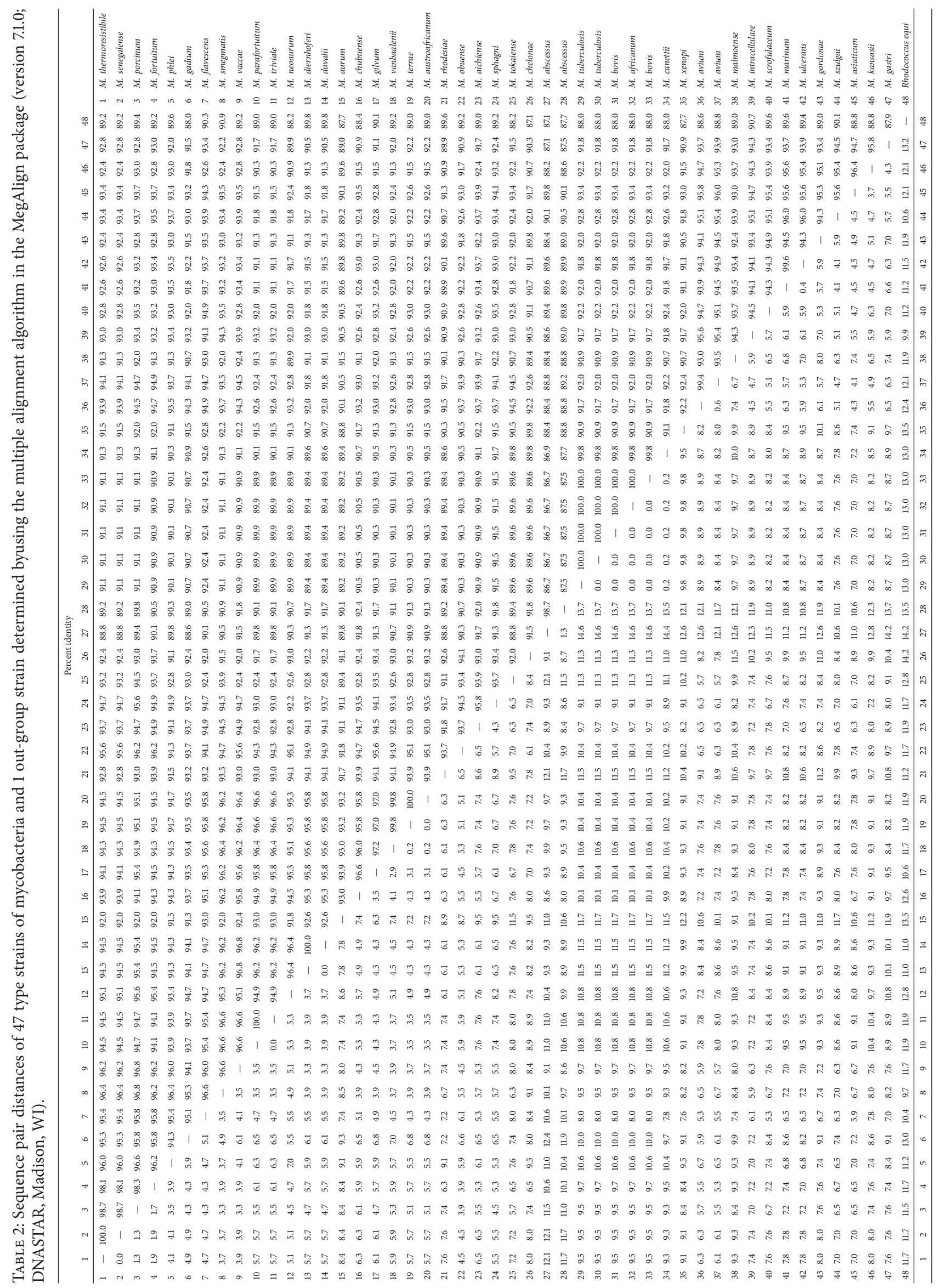




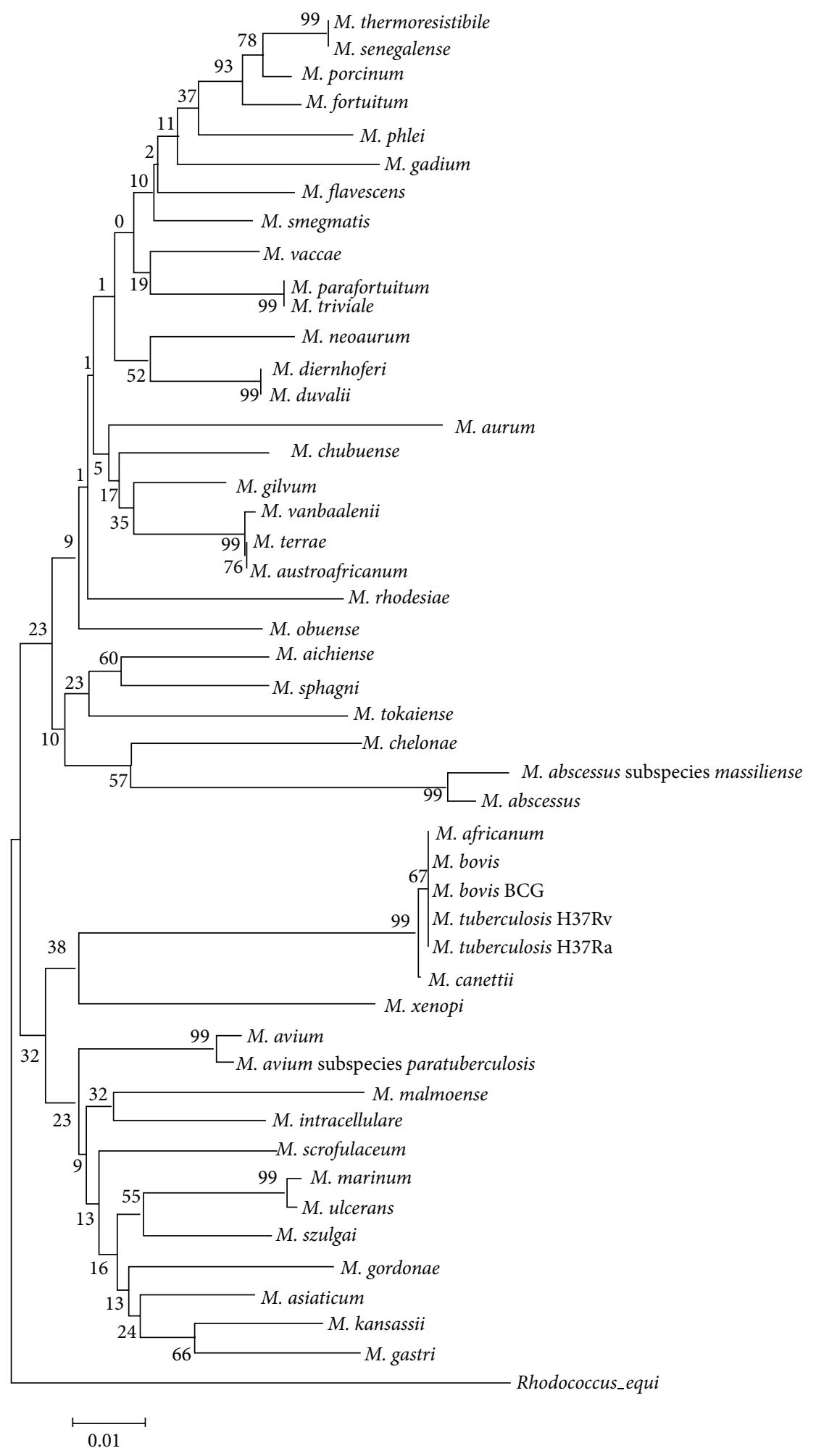

FIGURE 1: Phylogenetic tree based on $r p s A$ gene sequences shows the relationship of the 47 type strains of mycobacteria and 1 outgroup strain. This tree was constructed by the neighbor-joining method. Topology was also evaluated by bootstrap analysis (MEGA program, 1000 repeats, with $R$. equi as the outgroup). The numerical values in the tree represent bootstrap results. The distance between two strains is the sum of the branch lengths between them. 
TABLE 3: Comparison of mycobacteria identified by $16 \mathrm{~S}$ rDNA/ITS/hsp65/rpoB gene sequencing and by rpsA sequencing among the 188 clinical isolates ${ }^{\mathrm{a}}$.

\begin{tabular}{|c|c|c|c|c|}
\hline \multirow{3}{*}{ Primary identification ${ }^{\mathrm{b}}(n)$} & \multicolumn{4}{|c|}{ Primary identification $^{\mathrm{b}}(n)$} \\
\hline & \multicolumn{2}{|l|}{ First choice } & \multicolumn{2}{|l|}{ Second choice } \\
\hline & Identification $(n)$ & $\%$ match & Identification $(n)$ & $\%$ match \\
\hline \multirow{8}{*}{ M. intracellulare (75) } & M. intracellulare (4) & $100 \%$ & M. avium (4) & $95.6 \%$ \\
\hline & M. intracellulare (1) & $99.8 \%$ & M. avium (1) & $95.3 \%$ \\
\hline & M. intracellulare (53) & $98.9 \%$ & M. avium (53) & $95.1 \%$ \\
\hline & M. intracellulare (10) & $98.7 \%$ & M. avium (10) & $94.9 \%$ \\
\hline & M. intracellulare (1) & $98.5 \%$ & M. avium (1) & $95.3 \%$ \\
\hline & M. intracellulare (3) & $98.1 \%$ & M. avium (3) & $94.7 \%$ \\
\hline & M. intracellulare (1) & $97.5 \%$ & M. avium (1) & $93.2 \%$ \\
\hline & M. intracellulare (2) & $\mathbf{9 7 . 1 \%}$ & M. avium (2) & $96.1 \%$ \\
\hline \multirow{3}{*}{ M. abscessus (39) } & M. abscessus (9) & $100 \%$ & M. abscessus subspecies massiliense (9) & $98.7 \%$ \\
\hline & M. abscessus (10) & $99.8 \%$ & M. abscessus subspecies massiliense (10) & $98.5 \%$ \\
\hline & M. abscessus subspecies massiliense (20) & $99.4 \%$ & M. abscessus (20) & $99.1 \%$ \\
\hline \multirow{2}{*}{ M. kansasii (23) } & M. kansasii (22) & $100 \%$ & M. asiaticum (22) & $96.3 \%$ \\
\hline & M. szulgai (1) & $96.6 \%$ & M. marinum/M. ulcerans (1) & $96.0 \%$ \\
\hline \multirow{3}{*}{ M. avium (10) } & M. avium subspecies paratuberculosis (5) & $99.6 \%$ & M. avium (5) & $99.4 \%$ \\
\hline & M. avium subspecies paratuberculosis (4) & $99.8 \%$ & M. avium (4) & $99.2 \%$ \\
\hline & M. avium subspecies paratuberculosis (1) & $97.5 \%$ & M. avium (1) & $97.0 \%$ \\
\hline \multirow{5}{*}{ M. fortuitum complex (17) } & M. fortuitum (2) & $100 \%$ & M. senegalense/M. thermoresistibile (2) & $98.1 \%$ \\
\hline & M. fortuitum (11) & $99.8 \%$ & M. porcinum (11) & $98.5 \%$ \\
\hline & M. fortuitum (1) & $99.6 \%$ & M. porcinum (1) & $98.3 \%$ \\
\hline & M. fortuitum/M. porcinum (1) & $98.9 \%$ & M. senegalense/M. thermoresistibile (1) & $98.3 \%$ \\
\hline & M. thermoresistibile/M. senegalense (2) & $99.6 \%$ & M. fortuitum (2) & $98.1 \%$ \\
\hline \multirow{4}{*}{ M. gordonae (7) } & M. gordonae (1) & $99.2 \%$ & M. asiaticum (1) & $95.5 \%$ \\
\hline & M. gordonae (2) & $98.5 \%$ & M. asiaticum (2) & $96.3 \%$ \\
\hline & M. gordonae (3) & $95.9 \%$ & M. asiaticum (3) & $95.5 \%$ \\
\hline & M. szulgai (1) & $95.9 \%$ & M. gordonae (1) & $94.7 \%$ \\
\hline \multirow{3}{*}{ M. tuberculosis complex (16) } & M. tuberculosis (2) & $100 \%$ & M. canetti (2) & $99.8 \%$ \\
\hline & M. tuberculosis (12) & $99.8 \%$ & M. canetti (12) & $99.6 \%$ \\
\hline & M. tuberculosis (2) & $99.6 \%$ & M. canetti (2) & $99.4 \%$ \\
\hline M. neoaurum (1) & M. neoaurum (1) & $98.7 \%$ & M. smegmatis (1) & $95.4 \%$ \\
\hline
\end{tabular}

${ }^{a}$ Numbers in parentheses $(n)$ represent the numbers of isolates identified as a particular species.

${ }^{\mathrm{b}}$ Identification based on sequencing of at least two of the following: 16S rDNA, 16-23S rRNA gene internal transcribed spacer (ITS), and rpoB and hsp65 genes.

reference strains had similar homology with two or more species which suggests inadequate taxonomy within the currently described species. Based on nucleotide sequences of rpoB, hsp65, and sodA in clinical isolates of the Mycobacterium abscessus group, one-fourth of isolates had discordant identification [16]. Multilocus sequence typing and sequence analysis of several genes facilitate the identification of closely related species or subspecies [17].

In our study, we have demonstrated that the rpsA homology gene is a promising marker for mycobacterial species identification for both fast- and slow-growing mycobacteria. To be a good marker for species differentiation, the target gene should be stable and sequence variations should occur randomly; additionally, an extremely conserved or highly variable gene may not be adequate. As a single-copy housekeeping gene, $r p s A$ gene could work well as a target gene for
Mycobacterium species discrimination without ambiguous identification. The 16S rDNA gene has higher homology within the mycobacteria compared to the rpsA gene: $94.3 \%$ to $100 \%$ compared to $85.4 \%$ to $100 \%$. According to our work, when used as sole marker, $r p s A$ had better resolution than $16 s$ rDNA but had similar resolution as ITS, rpoB, and hsp65.

Due to the high resolution power of $r p s A$ for species identification, we presume it has potential of clinical use. From our own experience, we recommend that when performing species identification by homologue DNA sequence comparison, one should start with 16s RNA gene, plus at least one of the other markers such as ITS, rpoB, hsp65, and $\operatorname{rps} A$. When conflicting outcome or dubious outcomes yield, more markers should be added further. Even though 16s RNA gene is inferior to the other markers considering the capacity, it should be chosen firstly since 16s RNA gene has 
the most robust sequence database, which can help to avoid big identification errors due to unreliable database of other markers [18].

The main deterrents for the primary use of $r p s A$ sequencing as a routine means of identifying mycobacteria reside in the need for a comprehensive database. Therefore, we constructed our own rpsA sequence database by including as many type strains as possible and integrating $r p s A$ sequences deposited in GenBank such as M. avium subspecies paratuberculosis, M. ulcerans, M. vanbaalenii, M. abscessus subspecies massiliense, and $M$. canettii. Besides type strains, rpsA sequences of confirmed clinical strains were also included. We will constantly upgrade our database and the capability to identify the most recent described species.

\section{Conflict of Interests}

The authors declare that there is no conflict of interests regarding the publication of this paper.

\section{Authors' Contribution}

Hongfei Duan and Guan Liu contributed equally to this study.

\section{Acknowledgments}

The work was supported by the research funding from Infectious Diseases Special Project, Ministry of Health of China (2012ZX10003002-09), and Beijing Municipal Administration of Hospitals Clinical Medicine Development of Special Funding (ZYLX201304). All the type strains and clinical isolates used in this study were obtained from the "Beijing Bio-Bank of Clinical Resources on Tuberculosis (D09050704640000)," Beijing Chest Hospital.

\section{References}

[1] T. Adékambi, P. Berger, D. Raoult, and M. Drancourt, "rpoB gene sequence-based characterization of emerging nontuberculous mycobacteria with descriptions of Mycobacterium bolletii sp. nov., Mycobacterium phocaicum sp. nov. and Mycobacterium aubagnense sp. nov," International Journal of Systematic and Evolutionary Microbiology, vol. 56, no. 1, Article ID 63969, pp. 133-143, 2006.

[2] B. Ninet, M. Monod, S. Emler et al., "Two different 16S rRNA genes in a mycobacterial strain," Journal of Clinical Microbiology, vol. 34, no. 10, pp. 2531-2536, 1996.

[3] U. Reischl, K. Feldmann, L. Naumann et al., "16S rRNA sequence diversity in Mycobacterium celatum strains caused by presence of two different copies of 16S rRNA gene," Journal of Clinical Microbiology, vol. 36, no. 6, pp. 1761-1764, 1998.

[4] D. E. Griffith, T. Aksamit, B. A. Brown-Elliott et al., "An official ATS/IDSA statement: diagnosis, treatment, and prevention of nontuberculous mycobacterial diseases," American Journal of Respiratory and Critical Care Medicine, vol. 175, no. 4, pp. 367416, 2007.

[5] W. Shi, X. Zhang, X. Jiang et al., "Pyrazinamide inhibits transtranslation in Mycobacterium tuberculosis," Science, vol. 333, no. 6049, pp. 1630-1632, 2011.
[6] S. T. Cole, R. Brosch, J. Parkhill et al., "Deciphering the biology of Mycobacterium tuberculosis from the complete genome sequence," Nature, vol. 393, no. 6685, pp. 537-544, 1998.

[7] A. Roth, M. Fischer, M. E. Hamid, S. Michalke, W. Ludwig, and H. Mauch, "Differentiation of phylogenetically related slowly growing mycobacteria based on 16S-23S rRNA gene internal transcribed spacer sequences," Journal of Clinical Microbiology, vol. 36, no. 1, pp. 139-147, 1998.

[8] H. Ringuet, A. Honore, A. Varnerot et al., "hsp65 sequencing for identification of rapidly growing mycobacteria," Journal of Clinical Microbiology, vol. 37, no. 3, pp. 852-857, 1999.

[9] B.-J. Kim, S.-H. Lee, M.-A. Lyu et al., "Identification of mycobacterial species by comparative sequence analysis of the RNA polymerase gene (rpoB)," Journal of Clinical Microbiology, vol. 37, no. 6, pp. 1714-1720, 1999.

[10] J. Amita, T. Vandana, R. S. Guleria, and R. K. Verma, “Qualitative evaluation of mycobacterial DNA extraction protocols for polymerase chain reaction," Molecular Biology Today, vol. 3, no. 2, pp. 43-49, 2002.

[11] R. J. Pauls, C. Y. Turenne, J. N. Wolfe, and A. Kabani, "A high proportion of novel mycobacteria species identified by $16 \mathrm{~S}$ rDNA analysis among slowly growing AccuProbe-negative strains in a clinical setting," American Journal of Clinical Pathology, vol. 120, no. 4, pp. 560-566, 2003.

[12] G. E. Fox, J. D. Wisotzkey, and P. Jurtshuk Jr., "How close is close: 16S rRNA sequence identity may not be sufficient to guarantee species identity," International Journal of Systematic Bacteriology, vol. 42, no. 1, pp. 166-170, 1992.

[13] M. Drancourt, P. Berger, and D. Raoult, "Systematic 16S rRNA gene sequencing of atypical clinical isolates identified 27 new bacterial species associated with humans," Journal of Clinical Microbiology, vol. 42, no. 5, pp. 2197-2202, 2004.

[14] P. Kirschner, E. C. Bottger, D. T. Walton, and M. Valesco, "Microheterogeneity within rRNA of Mycobacterium gordonae," Journal of Clinical Microbiology, vol. 30, no. 4, pp. 10491050, 1992.

[15] C. Taillard, G. Greub, R. Weber et al., "Clinical implications of Mycobacterium kansasii species heterogeneity: swiss national survey," Journal of Clinical Microbiology, vol. 41, no. 3, pp. 12401244, 2003.

[16] E. Macheras, A.-L. Roux, F. Ripoll et al., "Inaccuracy of singletarget sequencing for discriminating species of the Mycobacterium abscessus group," Journal of Clinical Microbiology, vol. 47, no. 8, pp. 2596-2600, 2009.

[17] L. A. Almeida and R. Araujo, "Highlights on molecular identification of closely related species," Infection, Genetics and Evolution, vol. 13, no. 1, pp. 67-75, 2013.

[18] J. Dai, Y. Chen, and M. Lauzardo, "Web-accessible database of hsp65 sequences from Mycobacterium reference strains," Journal of Clinical Microbiology, vol. 49, no. 6, pp. 2296-2303, 2011. 

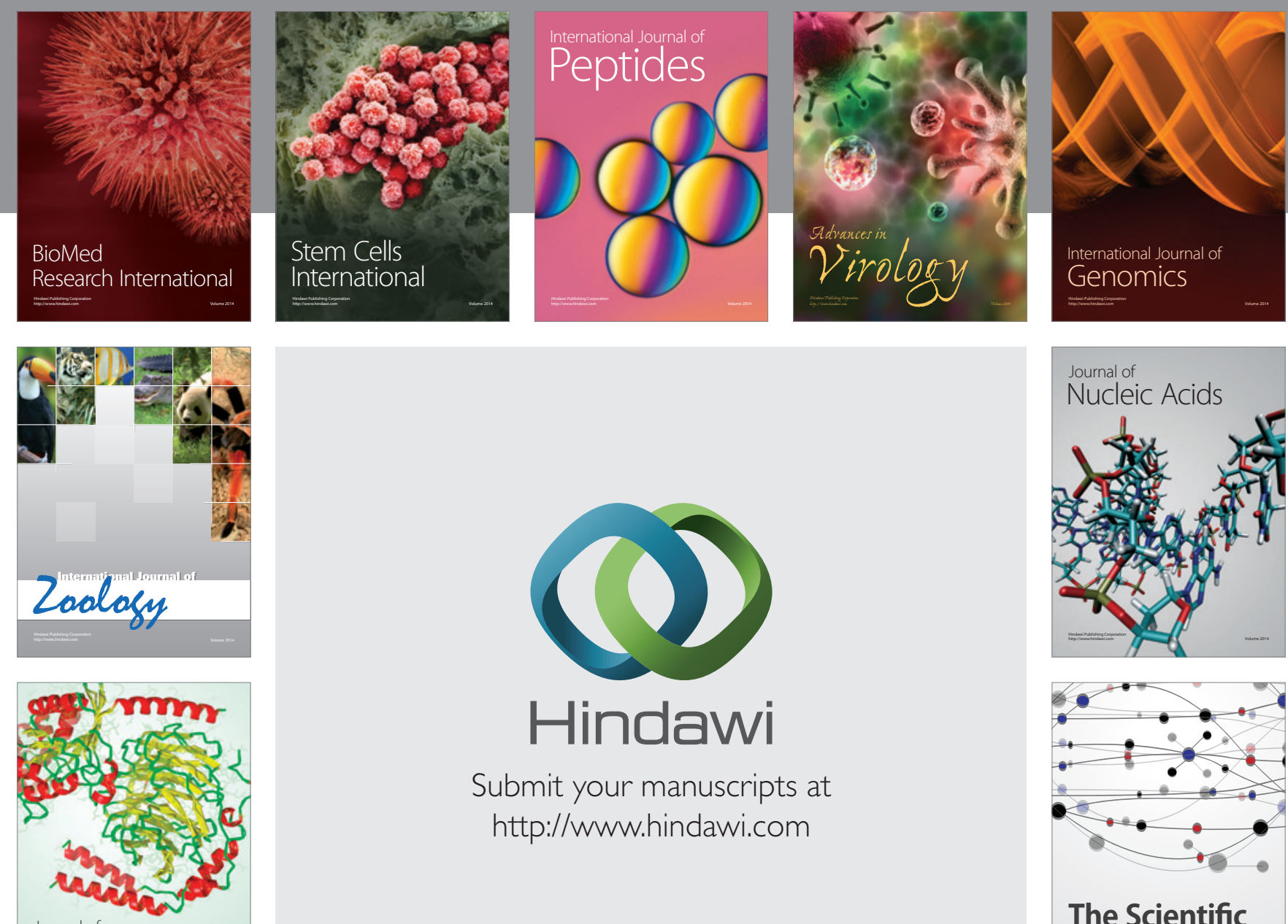

Submit your manuscripts at

http://www.hindawi.com

Journal of
Signal Transduction
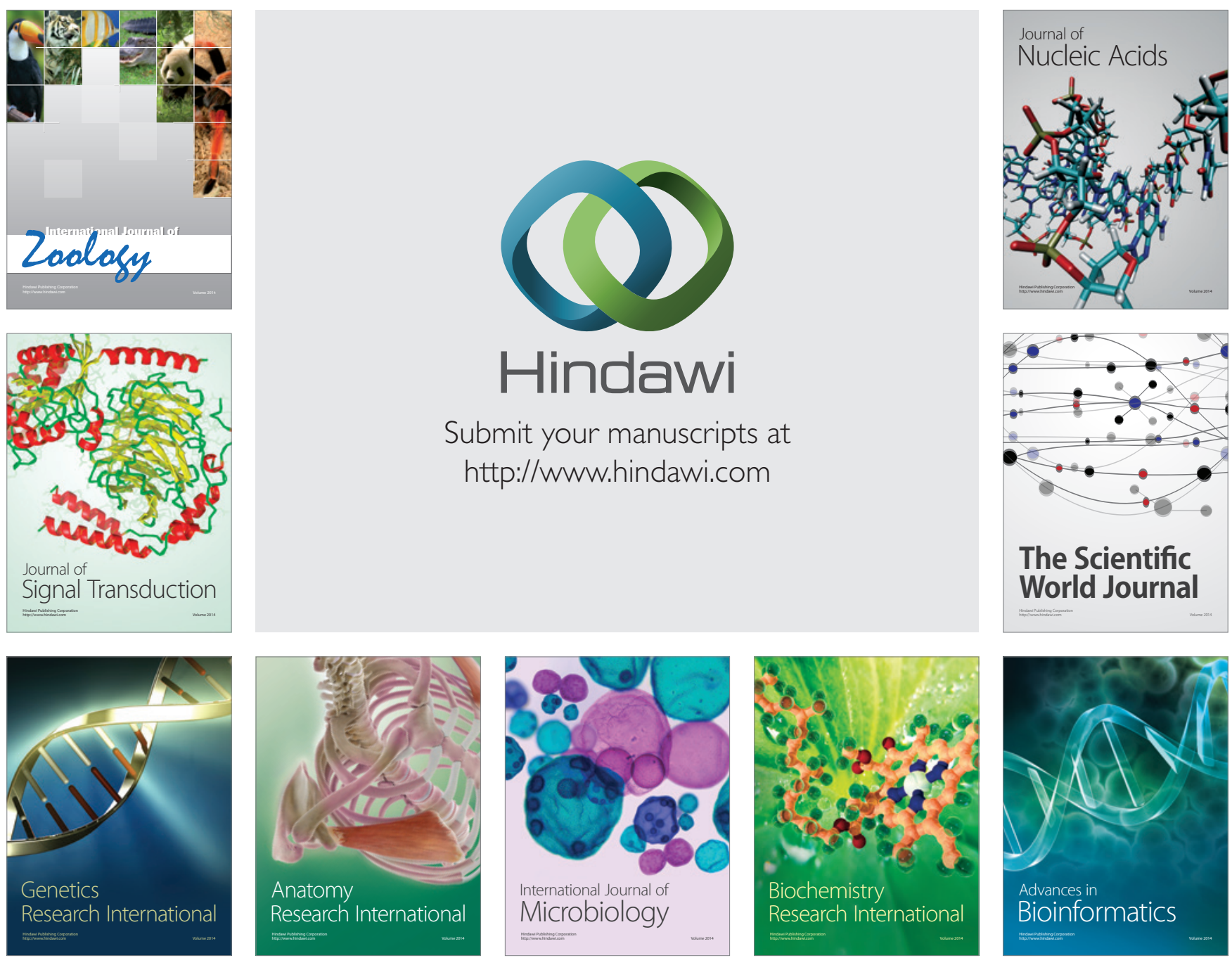

The Scientific World Journal
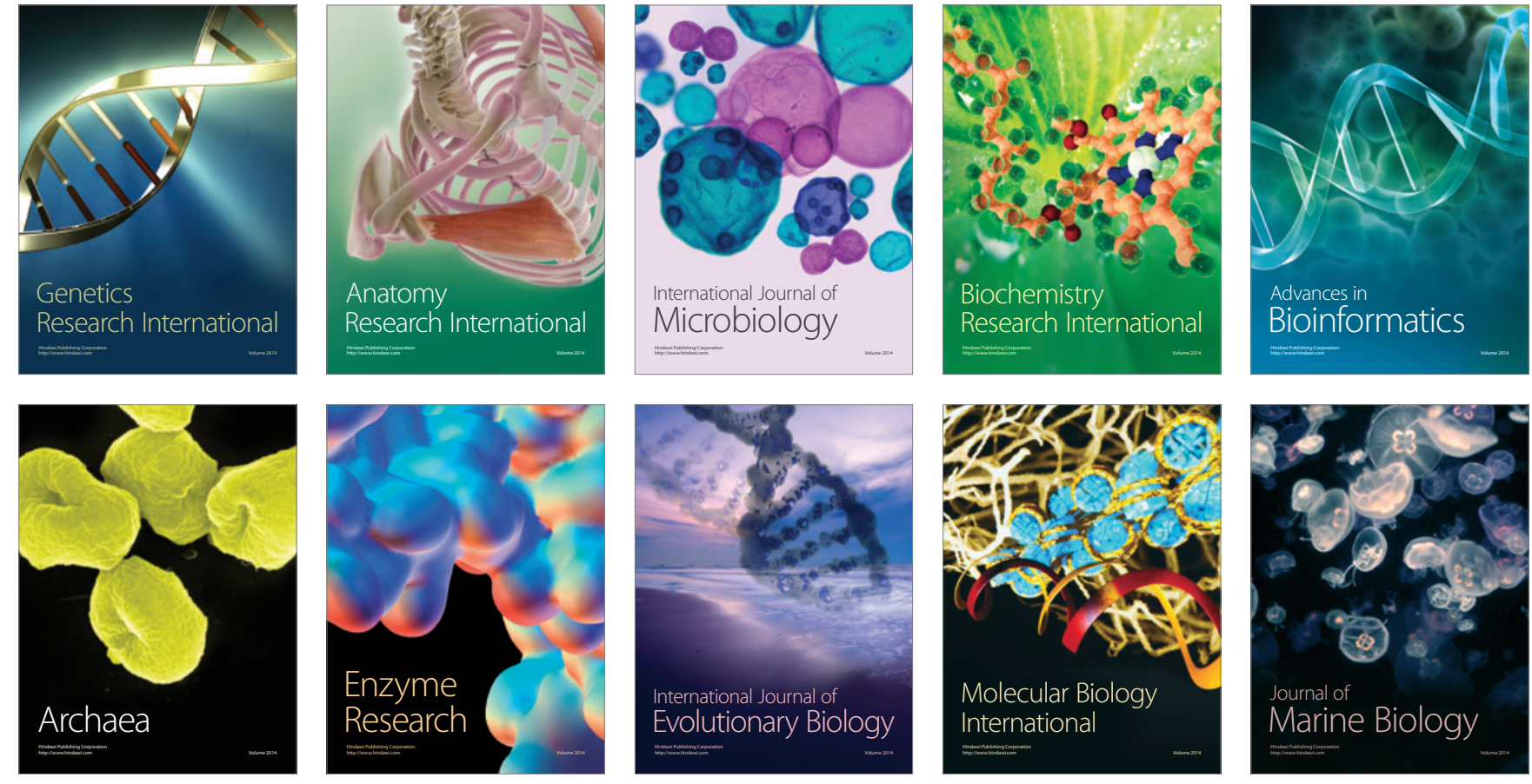